\title{
Construction of an miRNA-gene regulatory network in colorectal cancer through integrated analysis of mRNA and miRNA microarrays
}

\author{
JUN HU, XIN YUE, JIANZHONG LIU and DALU KONG \\ Department of Colorectal Cancer Surgery, National Clinical Research Center for Cancer, \\ Key Laboratory of Cancer Prevention and Therapy of Tianjin, Tianjin's Clinical Research Center for Cancer, \\ Tianjin Medical University Cancer Institute and Hospital, Tianjin 300060, P.R. China
}

Received December 19, 2017; Accepted August 8, 2018

DOI: $10.3892 / \mathrm{mmr} .2018 .9505$

\begin{abstract}
The aim of the present study was to identify potential biomarkers associated with colorectal cancer (CRC). The GSE32323 and GSE53592 mRNA and microRNA (miRNA) expression profiles were selected from the Gene Expression Omnibus database. The differentially expressed genes (DEGs) and differentially expressed miRNAs (DEMs) in CRC tissue samples compared with surrounding control tissue samples (DEGs-CC), and DEGs in cells treated with 5-aza-2'-deoxycitidine compared with untreated cells (DEGs-TC) were identified with the Limma package. The Database for Annotation, Visualization and Integrated Discovery was used to conduct the functional and pathways enrichment analysis. Differential co-regulation networks were constructed using the DCGL package of $R$. The targets of DEMs were identified using TargetScan. The overlaps between DEGs and the targets were selected. The miRNA-gene regulatory network of the overlaps was established. There were 145 DEMs, and 1,284 DEGs in DEGs-CC, and 101 DEGs in DEGs-TC. DEGs-CC were enriched in 196 Gene Ontology (GO) terms and 23 Kyoto Encyclopedia of Genes and Genomes (KEGG) pathways. DEGs-TC were enriched in $46 \mathrm{GO}$ terms and two KEGG pathways. A differential co-regulation network of the DEGs and a miRNA-gene regulatory network between DEMs and overlapped DEGs were respectively constructed. miR-124-3p, miR-145-5p and miR-320a may be critical in CRC, and serum/glucocorticoid regulated kinase 1 and SRY-box 9 may be potential biomarkers for CRC tumor progression.
\end{abstract}

Correspondence to: Dr Dalu Kong, Department of Colorectal Cancer Surgery, National Clinical Research Center for Cancer, Key Laboratory of Cancer Prevention and Therapy of Tianjin, Tianjin's Clinical Research Center for Cancer, Tianjin Medical University Cancer Institute and Hospital, 24 Huan-Hu-Xi Road, Ti-Yuan-Bei, He Xi, Tianjin 300060, P.R. China

E-mail: drkongdalu@163.com

Key words: colorectal cancer, bioinformatics, microRNA-gene regulatory network, differential co-regulation network

\section{Introduction}

Colorectal cancer (CRC) is a common malignancy that ranks as the second leading cause of cancer-associated mortality in men and women in the USA (1). Despite improvements in CRC therapy, CRC remains a major public health problem, and it is estimated that there are 1,000,000 individuals suffering from $\mathrm{CRC}$ worldwide, with the mortality rate is as high as $\sim 50 \%$ in certain developed countries (2). The tumor stage is the most important prognostic indicator for CRC. However, the tumors are often diagnosed at an intermediate or late stage, and the pathological staging cannot accurately predict recurrence (3). An immunochemical test has been used in CRC screening, which is considered more useful than colonoscopy in the Chinese population, and is less invasive and more accurate than colonoscopy $(4,5)$. The progression of CRC is a complex multigene, multistep, multistage process involving certain specific molecular alterations. A number of genes and pathways have been revealed to be involved the occurrence and development of CRC. For example, mutations of tissue inhibitor of metalloproteinases 2 and metalloproteinases are associated with the tumorigenesis and certain biological behaviors of CRC (6). The activation of $\mathrm{Wnt} / \beta$-catenin signaling occurs in the majority of cases of $\mathrm{CRC}$, and activation of this pathway is an early event in CRC tumorigenesis (7). However, the molecular mechanisms associated with $\mathrm{CRC}$ require further investigation, and it is important to identify novel biomarkers that may guide the diagnosis and therapy of CRC.

MicroRNAs (miRNAs) post-transcriptionally regulate the expression of numerous genes. miRNAs can silence gene expression by binding to the 3'untranslated regions (3'-UTRs) of a target mRNA, resulting in gene degradation or translation termination (8). Increasing evidence indicates that miRNAs are crucial in several types of cancer. miRNAs can regulate cell growth, cell death, cell proliferation and differentiation, in addition to tumorigenesis. Several signaling pathways and genes are reported as regulatory targets of miRNAs in cancer, including B-cell lymphoma 2 apoptosis regulator and sirtuin 1 genes in breast cancer (9), and KRas and Notch pathways in pancreatic cancer (10). Furthermore, miRNAs may also be useful as cancer biomarkers and treatment targets. However, 
the specific regulatory mechanism of miRNAs in CRC remains to be fully elucidated.

In the present study, bioinformatics methods were used to identify differentially expressed genes (DEGs) and differentially expressed miRNAs (DEMs) in CRC tissue samples compared with non-cancerous samples. The construction of the differential co-regulation network and miRNA-gene regulatory network may assist in improving current understanding of the regulatory mechanisms of miRNAs in CRC.

\section{Materials and methods}

Microarray data. The mRNA expression and miRNA profiles of the GSE32323 (11) and GSE53592 datasets were respectively downloaded from the Gene Expression Omnibus (GEO; http://www.ncbi.nlm.nih.gov/geo/) database. GSE53592 consisted of data from six samples, three CRC tissue samples and three surrounding control tissue. The miRNA expression profile was detected using the GPL8786 [miRNA-1_0] Affymetrix miRNA Array platform (Affymetrix, Inc., Santa Clara, CA, USA; http://www.affymetrix.com/analysis/index. affx). The mRNA dataset GSE32323 contained 44 samples, including 17 pairs of cancer and non-cancerous tissue samples from patients with $\mathrm{CRC}$, five pairs treated with 5-aza-2'-deoxycitidine and untreated cell line samples. Gene expression profiles were measured using the GPL570 [HG-U133_Plus_2] Affymetrix Human Genome U133 Plus 2.0 Array (Affymetrix, Inc.; http://www.affymetrix.com) platform.

Identification of DEMs and DEGs. For the mRNA dataset, the raw data were background corrected and normalized using the affy package version 1.58.0 (https://bioconductor.org/packages/release/bioc/html/affy.html) in $R$ version 2.10 .0 (12). If more than one probe corresponded to only one gene, the average expression value of these probes was considered as the expression value of the gene. The DEMs and DEGs in the CRC tissue samples compared with surrounding control tissue samples (DEGs-CC) and the DEGs in cell samples treated with 5-aza-2'-deoxycitidine compared with untreated samples (DEGs-TC) were identified using the limma package (http://bioconductor.org/packages/release/bioc/html/limma.

html) of $R$. The DEMs were identified according to the following criteria: false discovery rate (FDR) corrected P-value of $\mathrm{P}<0.05$ and $\mid \log _{2}$ (fold change) $\mid>1$. The screening criteria for the DEGs was $\mid \log _{2}$ (fold change) $\mid>1$ and Benjamini and Hochberg corrected $\mathrm{P}$-value of $\mathrm{P}<0.05$. Hierarchical clustering analysis of CRC tissue samples and non-cancerous samples based on the expression value of these DEGs was performed. The DEGs in cell samples treated with 5-aza-2'-deoxycitidine compared with untreated samples were identified via the limma package with the criteria of $\mathrm{P}<0.05$ and $\log _{2}$ (fold change) $\mid>0.5$.

Functional and pathway enrichment analysis. The Database for Annotation, Visualization and Integrated Discovery (DAVID; https://david.ncifcrf.gov/) is a widely used web-based tool for genomic functional annotations. To understand the biological functions of the DEGs, Gene Ontology (GO) terms and Kyoto Encyclopedia of Genes and Genomes (KEGG) pathway enrichment analyses were performed based on DAVID. The GO terms and KEGG pathways that contained more than two
Table I. Top 20 differentially expressed miRs in colorectal cancer tissue samples compared with surrounding control tissue samples.

\begin{tabular}{llr}
\hline miR ID & P-value & LogFC \\
\hline miR-195-5p & $3.62 \times 10^{-05}$ & -4.04 \\
miR-302c-5p & $1.52 \times 10^{-04}$ & 3.22 \\
miR-4328 & $2.71 \times 10^{-04}$ & -3.75 \\
miR-28-3p & $2.80 \times 10^{-04}$ & -4.90 \\
miR-186-5p & $3.11 \times 10^{-04}$ & -4.28 \\
miR-320a & $3.18 \times 10^{-04}$ & -2.85 \\
miR-30b-5p & $3.99 \times 10^{-04}$ & -3.68 \\
miR-101-3p & $5.02 \times 10^{-04}$ & -2.89 \\
miR-30c-5p & $5.30 \times 10^{-04}$ & -2.11 \\
miR-140-3p & $6.33 \times 10^{-04}$ & -2.42 \\
miR-145-5p & $7.54 \times 10^{-04}$ & -3.34 \\
miR-143-3p & $8.34 \times 10^{-04}$ & -5.10 \\
miR-378e & $8.40 \times 10^{-04}$ & -4.35 \\
miR-708-5p & $9.01 \times 10^{-04}$ & 4.26 \\
miR-125b-5p & $9.56 \times 10^{-04}$ & -3.67 \\
miRPlus-C1066 & $1.08 \times 10^{-03}$ & 3.12 \\
miR-320b & $1.11 \times 10^{-03}$ & -2.08 \\
miR-3158-5p & $1.29 \times 10^{-03}$ & 2.26 \\
miR-1973 & $1.31 \times 10^{-03}$ & -2.18 \\
miR-4748 & $1.49 \times 10^{-03}$ & 3.47 \\
\end{tabular}

miR, microRNA; FC, fold change.

genes were selected. $\mathrm{P}<0.05$ was used as the threshold to select the enriched GO terms and KEGG pathways.

Construction of the differential co-regulation network. DCGL is an $R$ package for screening differentially co-expressed genes (DCGs) and differentially co-expressed links (DCLs). It analyzes the expression correlation based on the exact co-expression changes to distinguish the significant co-expression changes and relatively minor ones. In the present study, differential co-regulation pairs were identified via DCGL version 2.1.2 in the CRC tissue samples compared with non-cancerous tissue samples, and the 5-aza-2'-deoxycitidine-treated cell line samples compared with the untreated cell line samples, and a differential co-regulation network was constructed based on the data.

Construction of the miRNA-gene regulatory network. The targets of the DEMs were identified based on the TargetScan version 6.2 (http://www.targetscan.org/) database. The overlaps between the DEGs and the targets of DEMs were selected. The miRNA-gene regulatory network was established and visualized using Cytoscape software version 3.1.0 (http://www. cytoscape.org/).

\section{Results}

DEGs and DEMs. A total of 145 DEMs were identified in the CRC samples compared with surrounding control tissue 

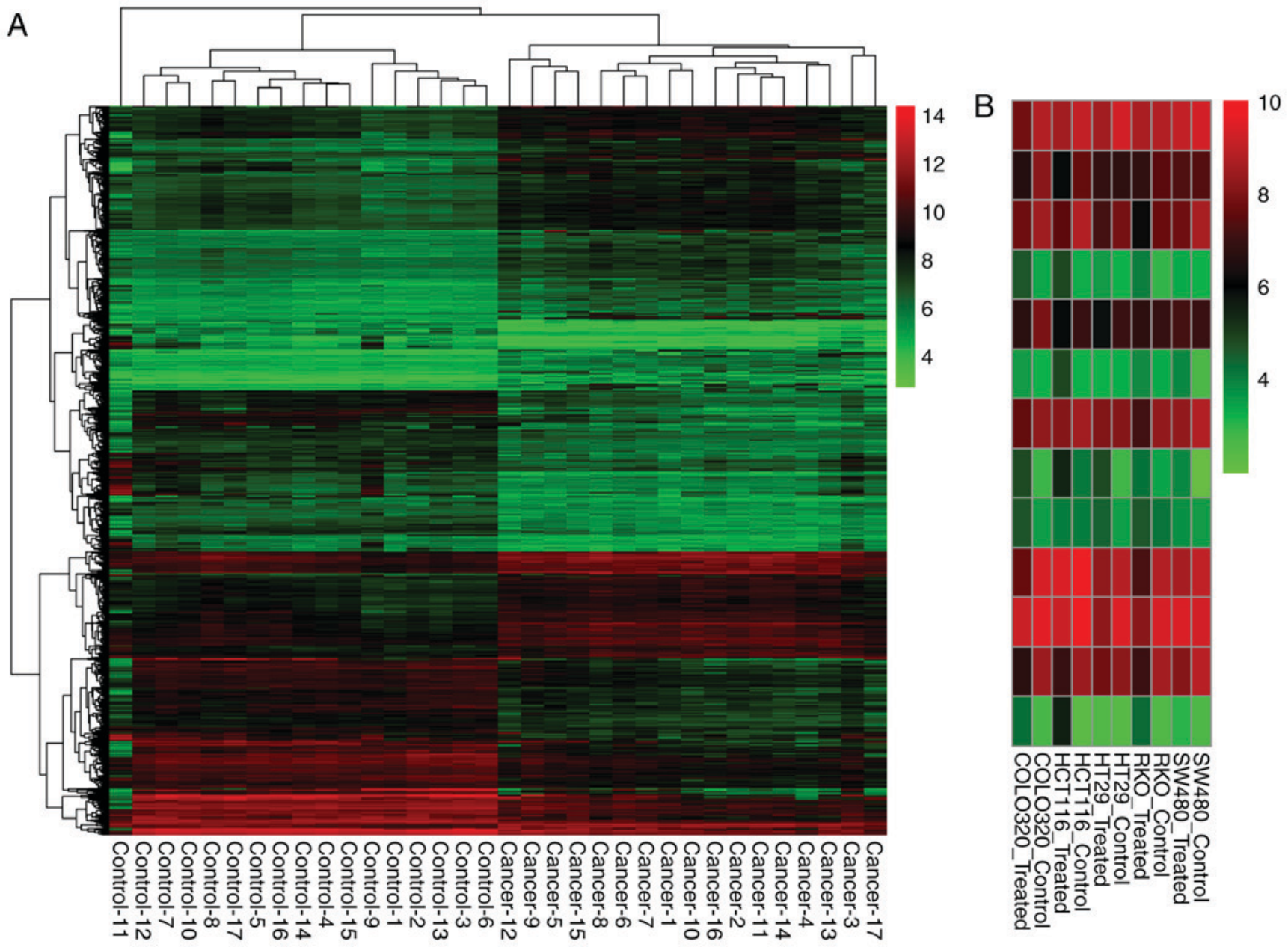

Figure 1. Identification of DEGs. (A) Cluster analysis of CRC tissue samples and non-cancerous tissue samples based on the DEGs-CC expression values. DEGs-CC separated cancer tissue samples from non-cancerous tissue samples. (B) Expression value heatmap of the overlaps between DEGs-CC and DEGs-TC in 5-aza-2'-deoxycitidine-treated cell line samples and untreated cell line samples. The color bar on the right represents the different expression values of DEGs. Overlaps separated treated cell line samples from untreated samples. CRC, colorectal cancer; DEGs, differentially expressed genes. DEGs-CC, DEGs in CRC tissue samples compared with non-cancerous tissue samples; DEGs-TC, DEGs in 5-aza-2'-deoxycitidine-treated cell line samples compared with untreated cell line samples.

samples. The top 20 DEMs according to the P-value are listed in Table I. Additionally, there were 1,284 DEGs-CC, including 638 downregulated DEGs and 646 upregulated DEGs. Cluster analysis of the CRC tissue samples and non-cancerous tissue samples based on the DEGs-CC expression values is shown in Fig. 1A. Furthermore, there were 101 DEGs-TC, including 42 downregulated DEGs and 59 upregulated DEGs. The top 20 DEGs in DEGs-CC and DEGs-TC are listed in Table IIA and Table IIB, respectively. There were 13 overlaps between the DEGs-CC and DEGs-TC. The expression value heatmap of these overlaps in the 5-aza-2'-deoxycitidine-treated cell line samples and untreated cell line samples is shown in Fig. 1B.

Enriched GO terms and pathways. The DEGs-CC were enriched in 196 GO terms and 23 KEGG pathways, and the DEGs-TC were enriched in 46 GO terms and two KEGG pathways. The top $10 \mathrm{GO}$ terms of DEGs-CC and DEGs-TC are listed in Table III according to P-value. Fig. 2 shows the enriched KEGG pathways of the DEGs-CC and DEGs-TC.

Differential co-regulation network. There were two types of association between the co-expression gene pairs and the regulatory factors. One is that the two co-expressed genes had a common transcription factor, and the co-expression association is not known or predicted by the transcription factor, gene regulation pairs (TF-bridged-DCL pairs). The other is that the co-expression pair is a known or predicted transcription factor, a gene regulation pair in itself (TF2target-DCL pairs). In the present study, 5,306 TF-bridged-DCL pairs and four TF2target-DCL pairs were obtained. The top 20 nodes according to degree are listed in Table IV.

miRNA-gene regulatory network. A total of 795 regulatory pairs between the DEMs and the DEGs were identified. Based on these pairs, the miRNA-gene regulatory network was constructed, which contained 451 nodes (Fig. 3). The top 20 nodes in the miRNA-gene network according to the degree are listed in Table V.

\section{Discussion}

The top two enriched GO terms of the DEGs-CC were 'mitotic nuclear division' and 'cell division', and were 'cell division' and 'G1/S transition of mitotic cell cycle' for the DEGs-TC. 
Table II. Top 20 DEGs-CC and DEGs-TC.

A, Top 20 DEGs-CC

\begin{tabular}{lcc}
\hline Gene & Adjusted P-value & LogFC \\
\hline ENC1 & $2.70 \times 10^{-12}$ & 2.548145 \\
CLDN1 & $2.70 \times 10^{-12}$ & 2.735442 \\
MMP28 & $1.87 \times 10^{-11}$ & -2.33266 \\
HILPDA & $1.87 \times 10^{-11}$ & 2.375653 \\
FOXQ1 & $1.87 \times 10^{-11}$ & 5.546198 \\
ASCL2 & $1.87 \times 10^{-11}$ & 2.24839 \\
SCARA5 & $2.88 \times 10^{-11}$ & -2.0155 \\
ABCA8 & $2.88 \times 10^{-11}$ & -1.263 \\
CHGA & $6.66 \times 10^{-11}$ & -1.98874 \\
C10orf54 & $7.64 \times 10^{-11}$ & -1.4338 \\
MYC & $8.02 \times 10^{-11}$ & 2.925688 \\
ST6GALNAC6 & $9.43 \times 10^{-11}$ & -2.36517 \\
DIEXF & $9.43 \times 10^{-11}$ & 1.409167 \\
SMPD1 & $1.03 \times 10^{-10}$ & -1.11622 \\
PLEKHA8P1 & $1.64 \times 10^{-10}$ & 1.146974 \\
ABI3BP & $1.72 \times 10^{-10}$ & -1.3326 \\
AJUBA & $2.03 \times 10^{-10}$ & 1.757746 \\
SLC11A2 & $2.47 \times 10^{-10}$ & 1.208803 \\
MTERF3 & $2.66 \times 10^{-10}$ & 1.755627 \\
SPIB & $2.92 \times 10^{-10}$ & -1.91248 \\
\hline
\end{tabular}

B, Top 20 DEGs-TC

\begin{tabular}{llc}
\hline Gene & P-value & LogFC \\
\hline GAGE3 & $5.09 \times 10^{-15}$ & 4.320948 \\
CXorf67 & 0.000186 & 0.866842 \\
MAEL & 0.000313 & 2.889106 \\
ACRC & 0.00077 & 4.065277 \\
TKTL1 & 0.000883 & 4.047408 \\
COX7B2 & 0.001184 & 1.485046 \\
PDIA2 & 0.001524 & 0.680444 \\
KIF11 & 0.002012 & -1.16205 \\
PAGE2B & 0.002021 & 2.791034 \\
CDC45 & 0.002089 & -0.93697 \\
AOC3 & 0.003546 & 1.582814 \\
DCAF4L1 & 0.003678 & 2.629287 \\
USHBP1 & 0.003725 & 0.529246 \\
NAA11 & 0.004706 & 0.730605 \\
SSX1 & 0.004742 & 1.379837 \\
ENTPD3-AS1 & 0.004955 & -0.53635 \\
DAZL & 0.005373 & 3.35696 \\
EID3 & 0.006215 & 0.708792 \\
OVAAL & 0.006445 & 1.746998 \\
TDRD12 & 0.006801 & 2.10315 \\
\hline
\end{tabular}

DEGs, differentially expressed genes; DEGs-CC, differentially expressed genes in CRC tissue samples compared with non-cancerous tissue samples; DEGs-TC, differentially expressed genes in 5-aza-2'-deoxycitidine-treated cell line samples compared with untreated cell line samples; FC, fold change.
These processes are critical in cancer development. Mitotic nuclear division is a key step in cell division, which is an essential process in tumorigenesis (13). Cell division increases the risk of various genetic errors. DNA adducts or other forms of single-stranded DNA damage are usually converse to gaps or mutations in the process of cell division (14). Tumor development is caused by the activation or altered expression of proto-oncogenes to oncogenes and the inactivation of tumor suppressor genes (15). Oncogenes can be activated by amplification translocation, or mutation. The loss or inactivation of tumor suppressor genes can promote cell division (16). Additionally, targeting the protein cell division cycle 7 kinase is a novel method for treating cancer. The G1/S transition in the mitotic cell cycle is a key step in which DNA replication is initiated. Deregulation of the cell cycle facilitates the aberrant cell proliferation that is characteristic of cancer (17). Cyclin D1 and cyclin-dependent kinase 4 are important regulators of the G1/S transition (18). The overexpression of cyclin D1 is reported to be involved in the development and progression of certain types of cancer, including esophageal and breast cancer (19-21). As a major regulator of the G1/S transition, cyclin D1 is an oncogenic driver in human cancer and may also serve as a therapeutic target (19). The top two enriched pathways for DEGs-CC were 'cell cycle' and 'mineral absorption', whereas the top two enriched pathways for DEGs-TC were 'cell cycle' and 'DNA replication'. The association between the cell cycle and cancer development is well established. In addition, the cell cycle phase can be used as a prognostic marker and therapeutic target in various types of cancer (22). DNA replication, a fundamental cellular process, is closely linked to cell proliferation. Inhibiting DNA replication-initiation proteins can induce the apoptosis of cancer cells (23). In CRC, DNA replication can serve as a prognostic biomarker in young patients with CRC. However, the association between mineral absorption and cancer development remains to be fully elucidated.

The top five genes in the differential co-regulation network were serum/glucocorticoid regulated kinase 1 (SGK1), SRY-box 9 (SOX9), solute carrier family 25 member 23 (SLC25A23), solute carrier family 20 member 1 (SLC20A1) and endothelin 3 (EDN3). SGK1 encodes a serine/threonine protein kinase, which is also associated with cell survival pathways (24). $S G K 1$ is also involved in the stimulation of motility, adhesiveness and invasiveness, and thus contributes to tumor metastasis (25). It has been reported that inhibition of the function of $S G K 1$ significantly decreased breast cancer cell adhesion, suggesting a role for $S G K 1$ in the aggressive phenotype of cancer cells (25). In CRC, $S G K 1$ was important for colorectal tumor growth and may be an attractive pharmacological target for cytostatic therapy. SOX9 is an important downstream factor induced by $\beta$-catenin (26). SOX9 is a high mobility group box transcription factor involved in embryonic development and required for differentiation, lineage commitment and epithelial-mesenchymal transition (27). High expression of SOX9 is associated with poor prognosis in various types of human cancer, including breast cancer, prostate cancer, melanomas and CRC (28-30). In CRC, a previous study reported that high expression of SOX9 was an independent poor risk factor in the prognosis of CRC, which may be used to predict clinical outcomes for patients with CRC (31). Another study demonstrated 
Table III. Top 10 GO terms of DEGs-CC and DEGs-TC.

\begin{tabular}{|c|c|c|c|}
\hline GO ID & GO term & Genes (n) & P-value \\
\hline \multicolumn{4}{|l|}{ DEGs-CC } \\
\hline GO:0007067 & Mitotic nuclear division & 46 & $6.21 \times 10^{-10}$ \\
\hline GO:0051301 & Cell division & 56 & $2.07 \times 10^{-09}$ \\
\hline GO:0000082 & G1/S transition of mitotic cell cycle & 22 & $2.93 \times 10^{-06}$ \\
\hline GO:0007062 & Sister chromatid cohesion & 21 & $1.27 \times 10^{-05}$ \\
\hline GO:0006260 & DNA replication & 26 & $3.27 \times 10^{-05}$ \\
\hline GO:0007052 & Mitotic spindle organization & 10 & $9.93 \times 10^{-05}$ \\
\hline GO:0045926 & Negative regulation of growth & 8 & $1.41 \times 10^{-04}$ \\
\hline GO:0008283 & Cell proliferation & 44 & $2.02 \times 10^{-04}$ \\
\hline GO:0042127 & Regulation of cell proliferation & 27 & $2.39 \times 10^{-04}$ \\
\hline GO:0006271 & DNA strand elongation involved in DNA replication & 7 & $2.55 \times 10^{-04}$ \\
\hline \multicolumn{4}{|l|}{ DEGs-TC } \\
\hline GO:0051301 & Cell division & 12 & $3.57 \times 10^{-07}$ \\
\hline GO:0000082 & G1/S transition of mitotic cell cycle & 7 & $5.74 \times 10^{-06}$ \\
\hline GO:0000731 & DNA synthesis involved in DNA repair & 5 & $1.64 \times 10^{-05}$ \\
\hline GO:0006260 & DNA replication & 7 & $6.20 \times 10^{-05}$ \\
\hline GO:0007062 & Sister chromatid cohesion & 6 & $9.07 \times 10^{-05}$ \\
\hline GO:0000732 & Strand displacement & 4 & $1.99 \times 10^{-04}$ \\
\hline GO:0007059 & Chromosome segregation & 5 & $2.29 \times 10^{-04}$ \\
\hline GO:0007067 & Mitotic nuclear division & 7 & $7.82 \times 10^{-04}$ \\
\hline GO:0007283 & Spermatogenesis & 8 & 0.001517 \\
\hline GO:0007076 & Mitotic chromosome condensation & 3 & 0.001938 \\
\hline
\end{tabular}

GO, Gene Ontology; DEGs-CC, differentially expressed genes in colorectal cancer tissue samples compared with non-cancerous tissue samples; DEGs-TC, differentially expressed genes in 5-aza-2'-deoxycitidine-treated cell line samples compared with untreated cell line samples.

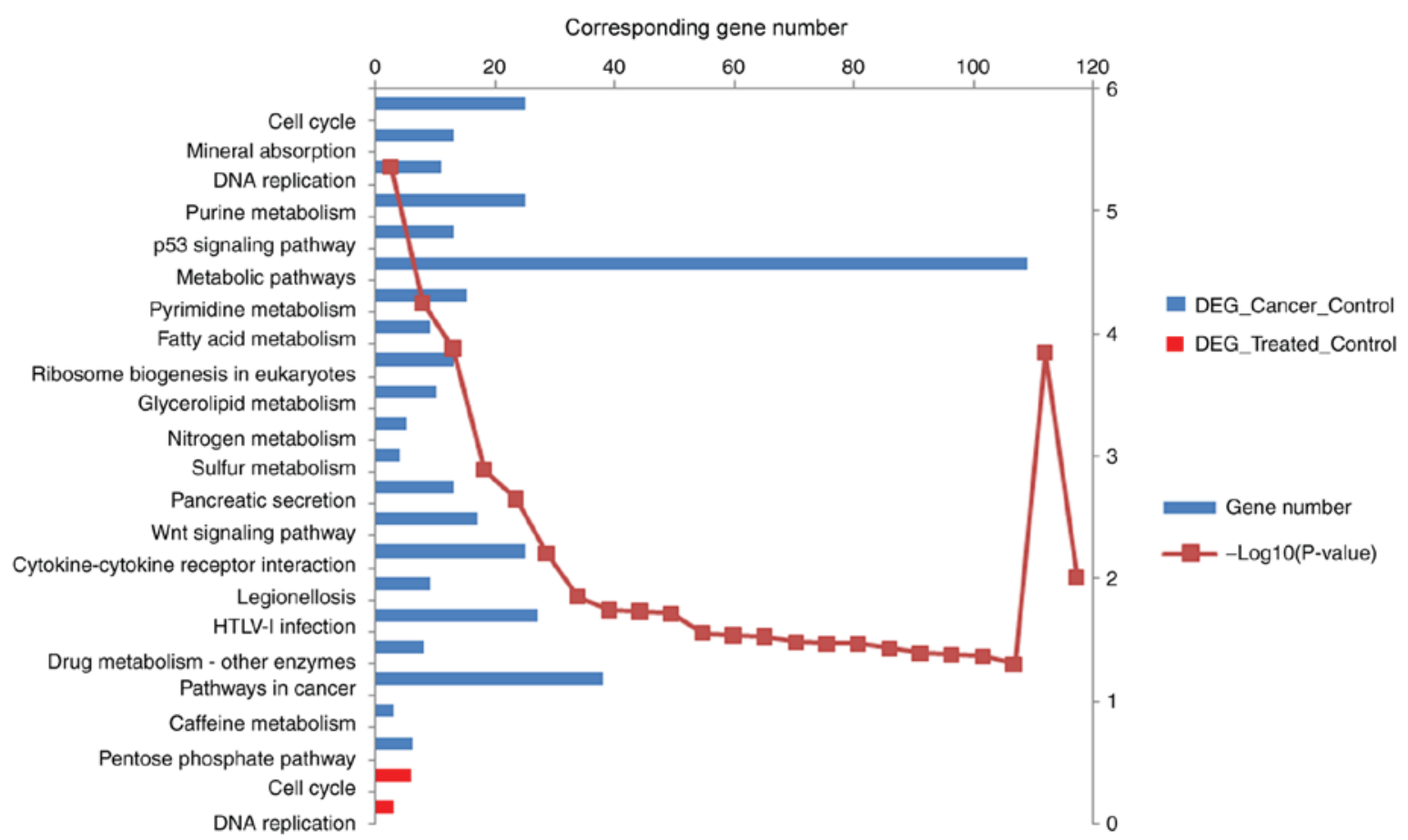

Figure 2. Enriched KEGG pathways for DEGs-CC and DEGs-TC, respectively. The blue bar represents the enriched KEGG of DEGs-CC, and the scale above represents its corresponding gene number; the red bar represents the enriched KEGG of DEGs-TC, and the scale above represents its corresponding gene number; the red fold line represents the $-\log _{10}$ (P-value) of the KEGG. DEGs-CC were enriched in 21 KEGG pathways, and DEGs-TC were enriched in two KEGG pathways. KEGG, Kyoto Encyclopedia of Genes and Genomes; DEGs, differentially expressed genes; DEGs-CC, DEGs in CRC tissue samples compared with non-cancerous tissue samples; DEGs-TC, DEGs in 5-aza-2'-deoxycitidine-treated cell line samples compared with untreated cell line samples. 
Table IV. Top 20 nodes in the differential co-regulation network.

\begin{tabular}{lc}
\hline Gene & Degree \\
\hline SGK1 & 842 \\
SOX9 & 615 \\
SLC25A23 & 557 \\
SLC20A1 & 479 \\
EDN3 & 424 \\
PRKAR2B & 255 \\
PAX5 & 250 \\
CDCA7 & 246 \\
WT1 & 231 \\
GPX2 & 227 \\
TPD52L1 & 203 \\
KLF9 & 181 \\
DAND5 & 178 \\
PSG1 & 178 \\
SP1 & 178 \\
C3orf70 & 177 \\
PIPOX & 162 \\
CREB1 & 161 \\
E2F & 152 \\
E2F1 & 152 \\
\hline
\end{tabular}

Table V. Top 20 nodes in the miR-gene regulatory network.

\begin{tabular}{lc} 
Node & Degree \\
\hline miR-124-3p & 94 \\
miR-101-3p & 75 \\
miR-186-5p & 74 \\
miR-145-5p & 65 \\
miR-320a & 51 \\
miR-374b-5p & 51 \\
miR-22-3p & 43 \\
miR-133b & 37 \\
miR-143-3p & 36 \\
miR-330-5p & 33 \\
miR-139-5p & 30 \\
miR-140-3p & 25 \\
miR-299-5p & 24 \\
miR-542-3p & 24 \\
miR-216a & 18 \\
miR-28-3p & 17 \\
miR-335-5p & 17 \\
miR-28-5p & 16 \\
miR-382-5p & 16 \\
miR-10a-5p & 16 \\
\hline
\end{tabular}

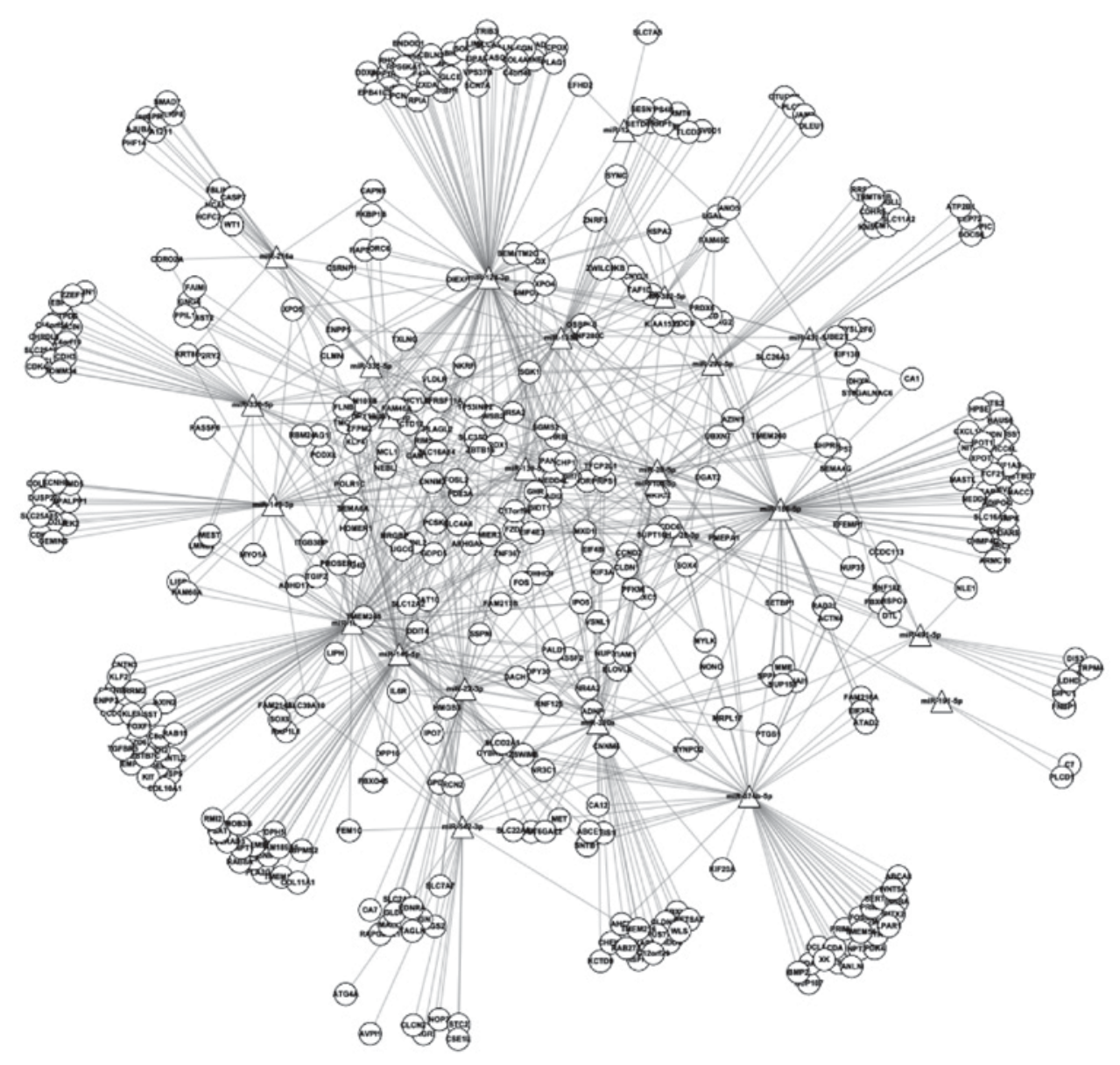

Figure 3. miRNA-gene regulatory network between DEMs and overlapped DEGs. The circles represent gene nodes; the triangles represent miRNA nodes; the lines represent miRNA-gene regulatory pairs. 795 regulatory pairs and 451 nodes were involved in the network. DEMs, differentially expressed miRNAs; DEGs, differentially expressed genes; miRNA, microRNA. 
that $S O X 9$ is important in the development, progression and metastasis of CRC, and that the combined detection of SOX9 and caudal type homeobox 2 may be useful as a marker to evaluate the degree of malignancy and the prognosis of CRC (32). Solute carrier family 25 member 23, solute carrier family 20 member 1 , and endothelin 3 are associated with cancer development (33-35). However, their specific roles in CRC require further investigation.

The top five nodes in the miRNA-gene regulatory network were miR-124-3p, miR-101-3p, miR-186-5p, $m i R-145-5 p$ and $m i R-320 a$. $m i R-124-3 p$ is a brain-enriched miRNA involved in the regulation of gastrulation and neural development. It functions as a tumor suppressor by targeting important genes, including Rac family small GTPase 1 (Rac1), sphingosine kinase 1, Rho-associated coiled-coil containing protein kinase 2 and enhancer of zeste 2 polycomb repressive complex 2 subunit. A previous study reported that the levels of $m i R-124-3 p$ were downregulated in breast cancer tissues, and that $m i R-124-3 p$ suppresses the expression of $\mathrm{Cbl}$ protooncogene, E3 ubiquitin protein ligase and negatively regulates the proliferation and invasion of breast cancer cells (36). In astrocytoma, $m i R-124-3 p$ has been shown to regulate cell proliferation, invasion, apoptosis and bioenergetics by targeting Pim-1 proto-oncogene, serine/threonine kinase (37). However, the regulatory the mechanism of $m i R-124-3 p$ in CRC remains to be fully elucidated. $m i R-320 a$ is considered to be a metastatic suppressor, and high expression is associated with improved outcomes in patients with CRC. Therefore, miR-320a may be an important suppressor of the development and metastasis of CRC. Several genes and pathways have been reported to be targets of $m i R-320 \mathrm{a}$. For example, a previous study demonstrated that $m i R-320 a$ suppresses the progression of CRC by targeting Rac1 (38). Another report showed that $m i R-320$ inhibits Wnt/ $\beta$-catenin signaling by targeting the 3 '-UTR of $\beta$-catenin (39). miR-145-5p is also a tumor suppressor miRNA, and it was previously demonstrated that $m i R-145$ was downregulated in certain types of cancer, including CRC, ovarian cancer and B-cell tumors (40-42). The downregulation of $m i R-145-5 p$ has been correlated with poor prognosis in patients with $\mathrm{CRC}$, and angiogenesis was inhibited in CRC by transfecting cancer cells with $m i R-145-5 p$ (43). $m i R-101-3 p$ and $m i R-186-5 p$ also regulated processes in human cancer $(44,45)$, although their roles in CRC remain to be fully elucidated.

In conclusion, bioinformatics analysis was used to identify important miRNAs and genes associated with the oncogenesis of CRC. miR-124-3p, miR-145-5p and miR-320a may be critical in regulating processes in CRC. $S G K 1$ and $S O X 9$ may be key genes that affect tumor progression of CRC. However, further investigations are required to further confirm this conclusion.

\section{Acknowledgements}

Not applicable.

\section{Funding}

No funding was received.

\section{Availability of data and materials}

All data generated or analyzed during this study are included in this article.

\section{Authors' contributions}

JH analyzed the microarray data. XY and JL performed functional and pathway enrichment analyses, and constructed the networks. DK designed the experiments and drafted the manuscript. All authors read and approved the final manuscript.

\section{Ethics approval and consent to participate}

Not applicable.

\section{Patient consent for publication}

Not applicable.

\section{Competing interests}

The authors declare that they have no competing interests.

\section{References}

1. Wu JT, Kakar S, Nelson RL, Mihalov ML, Hayward B, Gilbert PB and Ghosh L: Prognostic significance of DCC and p27Kip1 in colorectal cancer. Appl Immunohistochem Mol Morphol 13: 45-54, 2005.

2. Draht MX, Riedl RR, Niessen H, Carvalho B, Meijer GA, Herman JG, van Engeland M, Melotte V and Smits KM: Promoter $\mathrm{CpG}$ island methylation markers in colorectal cancer: The road ahead. Epigenomics 4: 179-194, 2012.

3. Marisa L, de Reyniès A, Duval A, Selves J, Gaub MP, Vescovo L, Etienne-Grimaldi MC, Schiappa R, Guenot D, Ayadi M, et al: Gene expression classification of colon cancer into molecular subtypes: Characterization, validation, and prognostic value. PLoS Med 10: e1001453, 2013.

4. Wong BC, Wong WM, Cheung KL, Tong TS, Rozen P, Young GP, Chu KW, Ho J, Law WL, Tung HM, et al: A sensitive guaiac faecal occult blood test is less useful than an immunochemical test for colorectal cancer screening in a Chinese population. Aliment Pharmacol Ther 18: 941-946, 2003.

5. Quintero E, Castells A, Bujanda L, Cubiella J, Salas D, Lanas Á, Andreu M, Carballo F, Morillas JD, Hernández C, et al: Colonoscopy versus fecal immunochemical testing in colorectal-cancer screening. N Engl J Med 366: 697-706, 2012.

6. Park KS, Kim SJ, Kim KH and Kim JC: Clinical characteristics of TIMP2, MMP2, and MMP9 gene polymorphisms in colorectal cancer. J Gastroenterol Hepatol 26: 391-397, 2011.

7. Aguilera O, Fraga MF, Ballestar E, Paz MF, Herranz M, Espada J, García JM, Muñoz A, Esteller M and González-Sancho JM: Epigenetic inactivation of the Wnt antagonist DICKKOPF-1 (DKK-1) gene in human colorectal cancer. Oncogene 25: 4116-4121, 2006

8. Pauli A, Rinn JL and Schier AF: Non-coding RNAs as regulators of embryogenesis. Nat Rev Genet 12: 136-149, 2011.

9. Li L, Yuan L, Luo J, Gao J, Guo J and Xie X: MiR-34a inhibits proliferation and migration of breast cancer through down-regulation of Bcl-2 and SIRT1. Clin Exp Med 13: 109-117, 2013.

10. Vorvis C, Koutsioumpa M and Iliopoulos D: Developments in miRNA gene signaling pathways in pancreatic cancer. Future Oncol 12: 1135-1150, 2016.

11. Khamas A, Ishikawa T, Shimokawa K, Mogushi K, Iida S, Ishiguro M, Mizushima $\mathrm{H}$, Tanaka $\mathrm{H}$, Uetake $\mathrm{H}$ and Sugihara $\mathrm{K}$ : Screening for epigenetically masked genes in colorectal cancer Using 5-Aza-2'-deoxycytidine, microarray and gene expression profile. Cancer Genomics Proteomics 9: 67-75, 2012.

12. Gautier L, Cope L, Bolstad BM and Irizarry RA: Affy-analysis of affymetrix GeneChip data at the probe level. Bioinformatics 20 : 307-315, 2004. 
13. Ames BN and Gold LS: Too many rodent carcinogens: Mitogenesis increases mutagenesis. Science 249: 970-971, 1990.

14. Ramel C: Short-term testing-are we looking at wrong endpoints? Mut Res 205: 13-24, 1988.

15. Stanbridge EJ: Identifying tumor suppressor genes in human colorectal cancer. Science 247: 12-13, 1990.

16. Preston-Martin S, Pike MC, Ross RK, Jones PA and Henderson BE: Increased cell division as a cause of human cancer. Cancer Res 50: 7415-7421, 1990.

17. Williams GH and Stoeber K: The cell cycle and cancer. J Pathol 226: 352-364, 2012.

18. Martín-Romero FJ, Santiago-Josefat B, Correa-Bordes J, Gutierrez-Merino $C$ and Fernandez-Salguero $P$ : Potassium-induced apoptosis in rat cerebellar granule cells involves cell-cycle blockade at the G1/S transition. J Mol Neurosci 15: 155-165, 2000.

19. Alao JP: The regulation of cyclin D1 degradation: Roles in cancer development and the potential for therapeutic invention. Mol Cancer 6: 24, 2007.

20. Bartkova J, Lukas J, Müller H, Lützhøt D, Strauss M and Bartek J: Cyclin D1 protein expression and function in human breast cancer. Int J Cancer 57: 353-361, 1994.

21. Jiang W, Zhang YJ, Kahn SM, Hollstein MC, Santella RM, Lu SH, Harris CC, Montesano R and Weinstein IB: Altered expression of the cyclin D1 and retinoblastoma genes in human esophageal cancer. Proc Natl Acad Sci USA 90: 9026-9030, 1993.

22. Diaz-Moralli S, Tarrado-Castellarnau M, Miranda A and Cascante M: Targeting cell cycle regulation in cancer therapy. Pharmacol Ther 138: 255-271, 2013.

23. Feng D, Tu Z, Wu W and Liang C: Inhibiting the expression of DNA replication-initiation proteins induces apoptosis in human cancer cells. Cancer Res 63: 7356-7364, 2003.

24. Brunet A, Park J, Tran H, Hu LS, Hemmings BA and Greenberg ME: Protein kinase SGK mediates survival signals by phosphorylating the forkhead transcription factor FKHRL1 (FOXO3a). Mol Cell Biol 21: 952-965, 2001.

25. Tangir J, Bonafé N, Gilmore-Hebert M, Henegariu O and Chambers SK: SGK1, a potential regulator of c-fms related breast cancer aggressiveness. Clin Exp Metastasis 21: 477-483, 2004.

26. Yano F, Kugimiya F, Ohba S, Ikeda T, Chikuda H, Ogasawara T, Ogata N, Takato T, Nakamura K, Kawaguchi $\mathrm{H}$ and Chung UI: The canonical Wnt signaling pathway promotes chondrocyte differentiation in a Sox9-dependent manner. Biochem Biophys Res Commun 333: 1300-1308, 2005.

27. Barrionuevo F and Scherer G: SOX E genes: SOX9 and SOX8 in mammalian testis development. Int J Biochem Cell Biol 42: 433-436, 2010.

28. Darido C, Buchert M, Pannequin J, Bastide P, Zalzali H, Mantamadiotis T, Bourgaux JF, Garambois V, Jay P, Blache $\mathrm{P}$, et al: Defective claudin-7 regulation by Tcf- 4 and Sox-9 disrupts the polarity and increases the tumorigenicity of colorectal cancer cells. Cancer Res 68: 4258-4268, 2008.

29. Passeron T, Valencia JC, Bertolotto C, Hoashi T, Le Pape E, Takahashi K, Ballotti R and Hearing VJ: SOX9 is a key player in ultraviolet B-induced melanocyte differentiation and pigmentation. Proc Natl Acad Sci USA 104: 13984-13989, 2007.

30. Wang H, Leav I, Ibaragi S, Wegner M, Hu GF, Lu ML, Balk SP and Yuan X: SOX9 is expressed in human fetal prostate epithelium and enhances prostate cancer invasion. Cancer Res 68: 1625-1630, 2008.
31. Lü B, Fang Y, Xu J, Wang L, Xu F, Xu E, Huang Q and Lai M: Analysis of SOX9 expression in colorectal cancer. Am J Clin Pathol 130: 897-904, 2008

32. Hu X, Yang W and Wu X: Expression of SOX9 and CDX2 in Colorectal Cancer. Chin J Surg Int Trad Western Med 17: 564-567, 2011 (In Chinese).

33. Schouten M, Fratantoni SA, Hubens CJ, Piersma SR, Pham TV, Bielefeld P, Voskuyl RA, Lucassen PJ, Jimenez CR and Fitzsimons CP: MicroRNA-124 and -137 cooperativity controls caspase-3 activity through BCL2L13 in hippocampal neural stem cells. Sci Rep 5: 12448, 2015.

34. Sato K and Akimoto K: Expression levels of KMT2C and SLC20A1 identified by information-theoretical analysis are powerful prognostic biomarkers in estrogen receptor-positive breast cancer. Clin Br Cancer 17: e135-e142, 2017.

35. Wiesmann F, Veeck J, Galm O, Hartmann A, Esteller M, Knüchel R and Dahl E: Frequent loss of endothelin-3 (EDN3) expression due to epigenetic inactivation in human breast cancer. Breast Cancer Res 11: R34, 2009.

36. Wang Y, Chen L, Wu Z, Wang M, Jin F, Wang N, Hu X, Liu Z, Zhang CY, Zen K, et al: miR-124-3p functions as a tumor suppressor in breast cancer by targeting CBL. BMC Cancer 16: 826, 2016.

37. Deng D, Wang L, Chen Y, Li B, Xue L, Shao N, Wang Q, Xia X, Yang Y and Zhi F: MicroRNA-124-3p regulates cell proliferation, invasion, apoptosis, and bioenergetics by targeting PIM1 in astrocytoma. Cancer Sci 107: 899-907, 2016.

38. Zhao H, Dong T, Zhou H, Wang L, Huang A, Feng B, Quan Y, Jin R, Zhang W, Sun J, et al: miR-320a suppresses colorectal cancer progression by targeting Racl. Carcinogenesis 35: 886-895, 2014.

39. Hsieh IS, Chang KC, Tsai YT, Ke JY, Lu PJ, Lee KH, Yeh SD, Hong TM and Chen YL: MicroRNA-320 suppresses the stem cell-like characteristics of prostate cancer cells by downregulating the Wnt/beta-catenin signaling pathway. Carcinogenesis 34: 530-538, 2013.

40. Li JM, Zhao RH, Li ST, Xie CX, Jiang HH, Ding WJ, Du P, Chen W, Yang M and Cui L: Down-regulation of fecal miR-143 and miR-145 as potential markers for colorectal cancer. Saudi Med J 33: 24-29, 2012.

41. Wu H, Xiao Z, Wang K, Liu W and Hao Q: MiR-145 is downregulated in human ovarian cancer and modulates cell growth and invasion by targeting p70S6K1 and MUC1. Biochem Biophys Res Commun 441: 693-700, 2013.

42. Xing AY, Wang B, Shi DB, Zhang XF, Gao C, He XQ, Liu WJ and Gao P: Deregulated expression of miR-145 in manifold human cancer cells. Exp Mol Pathol 95: 91-97, 2013

43. Thuringer D, Jego G, Berthenet K, Hammann A, Solary E and Garrido C: Gap junction-mediated transfer of miR-145-5p from microvascular endothelial cells to colon cancer cells inhibits angiogenesis. Oncotarget 7: 28160-28168, 2016.

44. Li CY, Xiong DD, Huang CQ, He RQ, Liang HW, Pan DH, Wang HL, Wang YW, Zhu HW and Chen G: Clinical value of miR-101-3p and biological analysis of its prospective targets in breast cancer: A study based on the cancer genome atlas (TCGA) and bioinformatics. Med Sci Monit 23: 1857-1871, 2017.

45. Li J, Xia L, Zhou Z, Zuo Z, Xu C, Song H and Cai J: MiR-186-5p upregulation inhibits proliferation, metastasis and epithelial-to-mesenchymal transition of colorectal cancer cell by targeting ZEB1. Arch Biochem Biophys 640: 53-60, 2018. 